\title{
General Cut-Generating Procedures for the Stable Set Polytope ${ }^{1}$
}

\author{
Ricardo C. Corrêa ${ }^{\mathrm{a}, *}$, Diego Delle Donne ${ }^{\mathrm{b}}$, Ivo $\mathrm{Koch}^{\mathrm{c}}$, Javier Marenco ${ }^{\mathrm{b}}$ \\ ${ }^{a}$ Universidade Federal Rural do Rio de Janeiro, Departamento de Ciência da Computação, Av. Governador \\ Roberto Silveira S/N, 26020-740 Nova Iguaçu - RJ, Brazil \\ ${ }^{b}$ Universidad Nacional de General Sarmiento, Instituto de Ciencias, J. M. Gutiérrez 1150, Malvinas Argentinas, \\ (1613) Buenos Aires, Argentina \\ ${ }^{c}$ Universidad Nacional de General Sarmiento, Instituto de Industria, J. M. Gutiérrez 1150, Malvinas Argentinas, \\ (1613) Buenos Aires, Argentina
}

\begin{abstract}
We propose general separation procedures for generating cuts for the stable set polytope, inspired by a procedure by Rossi and Smriglio and applying a lifting method by Xavier and Campêlo. In contrast to existing cut-generating procedures, ours generate both rank and non-rank valid inequalities, hence they are of a more general nature than existing methods. This is accomplished by iteratively solving a lifting problem, which consists of a maximum weighted stable set problem on a smaller graph. Computational experience on DIMACS benchmark instances shows that the proposed approach may be a useful tool for generating cuts for the stable set polytope.
\end{abstract}

\section{Introduction}

Let $G=(V, E)$ be an undirected graph with node set $V$ and edge set $E$. A stable set in $G$ is a subset of pairwise non-adjacent vertices of $G$. Given a graph $G$, the maximum stable set (MSS) problem asks for a stable set $S$ in $G$ of maximum cardinality. The stability number of $G$ is this maximum cardinality and is denoted by $\alpha(G)$. The MSS problem is computationally hard to solve in practice, being in NP-Hard unless the graph $G$ has some special structure. For an arbitrary input graph $G$, a number of exact methods have been developed to solve it through several combinatorial or mathematical programming-based techniques. For a survey of these theoretical and practical aspects of the MSS problem, see 3 and references therein.

Enumerative combinatorial algorithms have shown to be efficient to solve the MSS problem exactly for moderately sized graphs (for an overview, see [22]). Typically, such algorithms perform a search in a tree with the employment of simple and fast, but still effective, bounding procedures for pruning purposes. In this vein, the most successful approach involves the use of approximate

\footnotetext{
${ }^{1}$ This work has been partially supported by the Stic/AmSud joint program by CAPES (Brazil), CNRS and MAE (France), CONICYT (Chile) and MINCYT (Argentina) -project 13STIC-05- and the Pronem program by FUNCAP/CNPq (Brazil) - project ParGO.

*This author was with the ParGO research team, Universidade Federal do Ceará, Brazil, when most part of this work has been done.

Email addresses: correa@ufrrj.br (Ricardo C. Corrêa), ddelledo@ungs.edu.ar (Diego Delle Donne), ikoch@ungs.edu.ar (Ivo Koch), jmarenco@ungs.edu.ar (Javier Marenco)
} 
colorings of selected subgraphs of $\bar{G}$ (the complement of $G$ ). This bound is based on the following remark: if $\bar{G}$ admits an $\ell$-coloring, then $\alpha(G) \leq \ell$. This is a relatively weak bound and, consequently, the procedure to compute it is generally applied at numerous nodes of the search tree. However, it can be computed quickly by means of a greedy coloring heuristic implemented with bit parallelism operations [7, 18, 20].

An alternative to combinatorial algorithms is the use of sophisticated mathematical programming techniques to handle the combinatorial properties of the polytope associated with the formulation $\alpha(G)=\max \left\{\sum_{v \in V} x_{v} \mid x_{u}+x_{v} \leq 1, u v \in E, x_{v} \in\{0,1\}, v \in V\right\}$. Although combinatorial methods for the MSS problem from the literature outperform mathematical programming-based algorithms devised so far, it is of great interest to continue the search for efficient polyhedral methods for this problem. Despite its natural theoretical relevance, there are other motivations of algorithmic nature, namely: (a) the algorithms can be easily extended to the weighted version of the MSS problem, (b) MSS constraints frequently appear as a sub-structure in many combinatorial optimization problems, (c) in many situations, probing strategies gives MSS valid inequalities on conflicting variables for general mixed integer programs (see, e.g., 1, 4), and (d) real applications may need specific versions of the MSS problem with additional constraints. In this context, procedures for valid inequalities generation often turns out to be effective.

There are two main directions of research when polyhedral techniques, in particular procedures for valid inequalities generation, are concerned. The first direction is usually referred to as the lift-and-project method [6], which consists in three steps: first, a lifting operator is applied to the initial formulation to obtain a lifted formulation in a higher dimensional space; second, the lifted formulation is strengthened by means of additional valid inequalities; and third, a strengthened relaxation of the initial formulation is finally obtained as a result of an appropriate projection of the strengthened lifted formulation onto the original space. Several upper bounds for $\alpha(G)$ have been stated in connection of this method, such as the ones based on semidefinite programming (SDP, for short) relaxations described in [8, 13, which can be rather time-consuming to compute in practice. More recently, a new relaxation was introduced in [10] which preserves some theoretical properties of SDP relaxations in generating effective cuts but is computationally more tractable for a range of synthetic instances.

The second direction of research is integer programming, which in turn have followed two main approaches. The first approach consists in developing strong cuts coming from facet-inducing inequalities associated with special structures in the input graph (for instance, cliques, odd holes, webs, among others) and searching for specialized separation techniques for these families of inequalities (an up to date list of references for this approach can be found in [16]). The second approach relies on general cut-generating procedures which, starting from a fractional solution, search for a violated inequality with no prespecified structure. Such procedures were either shown to be effective in practice [16, 17] or to generate provably strong inequalities 23. The main contribution of this work are general procedures that are both effective and generate inequalities that can be proved to be facet-inducing under quite general conditions.

We now discuss existing works following the second approach, i.e., procedures generating cuts with no prespecified structure. Mannino and Sassano [14 introduced in 1996 the idea of edge projections as a specialization of Lovász and Plummer's clique projection operation [12. Many properties of edge projections are discussed in [14 and, based on these properties, a procedure computing an upper bound for the MSS problem is developed. This bound is then incorporated in a branch and bound scheme. Rossi and Smriglio take these ideas into an integer programming environment in [17, where a separation procedure based on edge projection is proposed. Finally, 
Pardalos et al. [16] extend the theory of edge projection by explaining the facetness properties of the inequalities obtained by this procedure. The authors give a branch and cut algorithm that uses edge projections as a separation tool, as well as several specific families of valid inequalities such as the odd hole inequalities (with a polynomial-time exact separation algorithm), the clique inequalities (with heuristic separation procedures), and mod- $\{2,3,5,7\}$ cuts.

Rossi and Smriglio propose in [17 to employ a sequence of edge projection operations to reduce the original graph $G$ and make it denser at the same time, allowing for a faster identification of clique inequalities on the reduced graph $G^{\prime}$. This procedure iteratively removes and projects edges with certain properties, and heuristically finds violated rank inequalities (i.e., inequalities of the form $\sum_{v \in A} x_{v} \leq \alpha(G[A])$, where $A \subseteq V$ and $G[A]$ is the subgraph of $G$ induced by $A$ ). A key step for achieving this is to be able to establish how $\alpha(G)$ is affected by these edge projections, or, in other words, how exactly $\alpha(G)$ relates to $\alpha\left(G^{\prime}\right)$. We aim at generalizing Rossi and Smriglio's procedure by projecting cliques instead of edges, so we also need to show how $\alpha(G)$ changes as a result of this operation. Our method allows thus to establish a more general relation between $G$ and the graph resulting from the clique projection.

In this article we propose the use of clique projections as a general method for cutting plane generation for the MSS, along with new clique lifting operations that lead to stronger inequalities than those obtained with the edge projection method. The proposed method is able to generate both rank and weighted rank valid inequalities (to be defined below), by resorting to the general lifting operation introduced in [23. This approach allows to produce cuts of a quite general nature, including cuts from the known families of valid inequalities for the MSS polytope. Based upon the projection and lifting operations, we give a separation procedure that departs from the usual template-based paradigm for generating cuts, and seeks to unify and generalize the separation procedures for the known cuts. In this sense, our main goal is to provide a more complete understanding of the maximum stable set polytope, which may help also in the solution of other combinatorial optimization problems. Experimental results are provided to validate the general procedure we propose.

This work is organized as follows. In Section 2 we define the MSS polytope $S T A B(G)$, we define the operation of clique projection and we explore some basic facts on this operation. Section 3 introduces the crucial concept of clique lifting, based on the results in 23. In Sections 4 and 5 we introduce our cut-generating method, by applying the lifting method presented in [23]. Finally, in Section 6 we present some computational experience on the DIMACS and randomly generated instances, which show that the method is competitive. The paper is closed with some concluding remarks in Section 7

\section{The Stable Set Polytope and the Clique Projection Operation}

Let $n:=|V|, N_{G}(v)$ be the neighborhood of $v$ in graph $G$, and $\mathcal{S}(G) \subseteq\{0,1\}^{n}$ be the set of all characteristic vectors of stable sets of $G$. We write simply $N(v)$ and $\mathcal{S}$, respectively, when $G$ is clear from context. For $W \subseteq V, \mathcal{S}(G[W])$ stands for the characteristic vectors of stable sets of $G$ involving vertices in $W$ only. The polytope of stable sets of $G$ is denoted by

$$
\operatorname{STAB}(G)=\operatorname{conv}\{x \mid x \in \mathcal{S}(G)\} .
$$

Note that the stability number of $G$ is $\alpha(G)=\max \left\{\sum_{v \in V} x_{v} \mid x \in S T A B(G)\right\}$. If $c \in \mathbb{R}^{n}$, then the weighted stability number of $G$, according to $c$ is $\alpha(G, c)=\max \left\{c^{\top} x \mid x \in S T A B(G)\right\}$. The 
general form of a facet-inducing inequality of $S T A B(G)$ is

$$
c^{\top} x \leq \alpha(G[H], c),
$$

where $c \in \mathbb{R}^{n}, c \geq \mathbf{0}, H=\left\{v \in V \mid c_{v}>0\right\}$, and $(G[H], c)$ is a so-called facet-subgraph of $G$ [11. Note that if $c \in\{0,1\}^{n}$ then we have the rank inequality mentioned in the Introduction.

Our interest is to build inequalities of type (1) by means of the following operation.

Definition 1 (Clique Projection [12]). Let $W \subseteq V,|W| \geq 2$, be a clique in $G$. The clique projection of $W$ gives the graph $G \mid W=(V, E \mid W)$ in which $E \mid W=E \cup\{u v \notin E \mid W \subseteq N(u) \cup N(v)\}$.

Figure 1 shows an example of this operation. The edges in $(E \mid W) \backslash E$ (i.e., the added edges after the projection) are called false edges. These are the edges simulated by $W$ in the sense stated in the lemma below. For $W \subseteq V$, we define $x_{W}=\sum_{v \in W} x_{v}$.

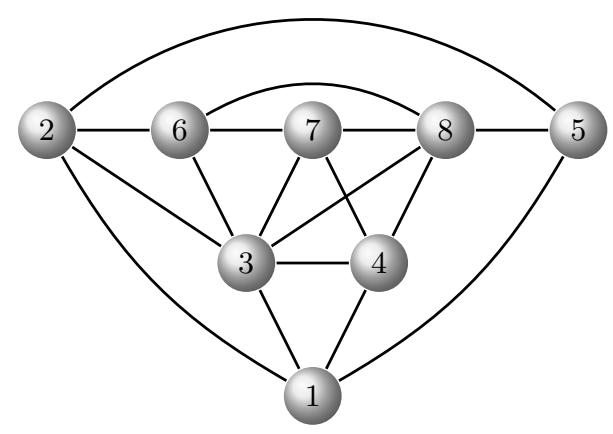

(a) Graph $G$.

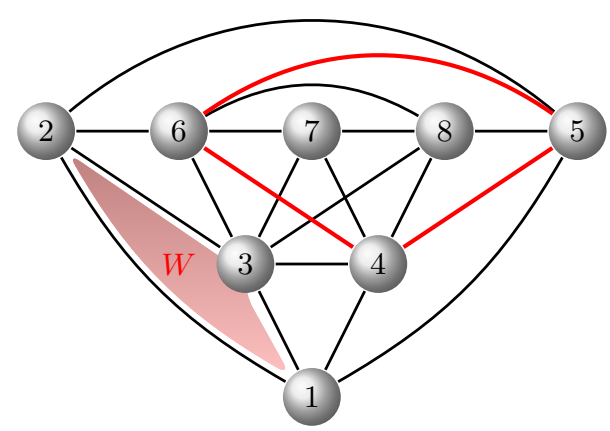

(b) Projected graph $G \mid W$.

Figure 1: Projected graph $G \mid W$ (with 3 false edges) is obtained after projecting $W=\{1,2,3\}$.

Lemma 1. $F_{W}=\left\{x \in S T A B(G) \mid x_{W}=1\right\} \subseteq S T A B(G \mid W) \subseteq S T A B(G)$.

Proof. For the first inclusion, we show that $x \in(S T A B(G) \backslash S T A B(G \mid W)) \cap \mathcal{S}(G)$ implies $x \notin F_{W}$. For such an $x$, there is a false edge $w z \in(E \mid W) \backslash E$ such that $x_{\{w\}}=x_{\{z\}}=1$. By definition of clique projection, $W \subseteq N(w) \cup N(z)$. Hence, for every $v \in W$, either $v \in N(w)$ or $v \in N(z)$ holds, leading to $x_{W}=0$. The second inclusion stems directly from the definition of clique projection.

A clique projection of an edge $u v$ is also referred to as edge projection. This term is employed in [16, 17] with a slightly different meaning since, in those papers, the vertices in $(N(u) \cap N(v)) \cup$ $\{u, v\}$ are removed when performing the projection. A fundamental property of edge projection is the following.

Definition 2 ([17]). An edge $u v \in E$ is projectable in $G$ if there exists a maximum stable set $S$ in $G$ such that $S \cap\{u, v\} \neq \emptyset$.

Lemma 2 ([14]). If $u v \in E$ is a projectable edge in $G$, then $\alpha(G)=\alpha(G \mid\{u, v\})$.

Proof. Since $u v$ is projectable, we get $\alpha(G) \leq \alpha(G \mid\{u, v\})$. On the other hand, $\alpha(G) \geq \alpha(G \mid$ $\{u, v\})$ due to Lemma 1 . 
Results presented in 14, 17 yield that if $N(u)-\{v\}$ is a clique, then $u v$ is projectable in every induced subgraph of $G$ containing $u$ and $v$. Indeed, in such a situation the projection of $u v$ is equivalent to the projection of the clique $\{u, v\} \cup(N(u) \cap N(v))$. Thus, define the subgraph $\tilde{G}$ as the graph obtained from $G$ by removing the edges connecting $u$ to all the vertices in $N(u) \backslash W$, where $W \subseteq N(u)-\{v\}$ induces a clique in $G$. Lemma 2 can then be used to write

$$
\alpha(\tilde{G} \mid\{u, v\}[H])=\alpha(\tilde{G}[H]) \geq \alpha(G[H]),
$$

for every $H \subseteq V$ such that $\{u, v\} \subseteq H$. A direct consequence is that the rank inequality $x_{H} \leq$ $\alpha(\tilde{G} \mid\{u, v\}[H])$, valid for $\tilde{G} \mid\{u, v\}$, is also valid for $G$.

\section{The Clique Lifting Operation}

In this section we lay a lifting operation that can be applied to valid inequalities of a projected graph to obtain valid inequalities for $S T A B(G)$. Given an inequality

$$
c^{\top} x=\sum_{v \in H} c_{v} x_{v} \leq \beta
$$

with $H \subseteq V, \beta \in \mathbb{R}$, and $c \in \mathbb{R}^{n}$ such that $c_{v} \neq 0$ if and only if $v \in H$, we say that $H$ is the support of (2). We are now in position of stating the lifting lemma on which our cut-generating procedure is based. For ease of presentation, we will restrict ourselves to a simplified version of this result in terms of the stable set polytope, and we refer the reader to [23] for the general result. In order to keep this work self-contained, we also provide a proof of this simplified version.

Lemma 3 (Simplified version of the Lifting Lemma [23]). Let $W \subseteq V$ be a clique of $G$. If $c^{\top} x \leq d$, $c \in \mathbb{R}^{n}$ and $d \in \mathbb{R}$, is a valid inequality for $F_{W}=\left\{x \in S T A B(G) \mid x_{W}=1\right\}$ with support $H \subseteq V$, then

$$
f(x)=\left(c^{\top} x-d\right)-\lambda\left(x_{W}-1\right) \leq 0,
$$

with the lifting factor $\lambda$ being such that

$$
\lambda \leq d-\alpha(G[H \backslash W], c),
$$

is a valid inequality for $S T A B(G)$. In addition, if $W$ is a maximal clique, $c^{\top} x \leq d$ is facet-defining for $F_{W}$, and $\lambda$ satisfies (4) at equality, then (3) is facet-defining for $S T A B(G)$.

Proof. To prove validity, it is sufficient to show that (3) holds for any $x \in \mathcal{S}$. If $x \in F_{W}$, then $f(x) \leq 0$ holds because $c^{\top} x \leq d$ is valid for $F_{W}$. Otherwise, $x_{W}=0$ and, by definition,

$$
f(x)=\left(c^{\top} x-d\right)+\lambda \leq c^{\top} x-\alpha(G[H \backslash W], c) \leq 0 .
$$

Now, assume that $W$ is a maximal clique (so $F_{W}$ is a facet of $S T A B(G)$ ) and $c^{\top} x \leq d$ is facetdefining for $F_{W}$, and let $x^{1}, \ldots, x^{n-1}$ be $n-1$ affinely independent vectors in $\left\{x \in F_{W} \mid c^{\top} x=d\right\}$. Clearly, $f\left(x^{i}\right)=0$, for all $i \in\{1, \ldots, n-1\}$. Additionally, let $x^{n}$ be the characteristic vector of a maximum weight independent set of $G[H \backslash W]$ according to $c$. It stems from $\lambda=d-\alpha(G[H \backslash W], c)$ that

$$
f\left(x^{n}\right)=\left(c^{\top} x^{n}-d\right)-\lambda\left(x_{W}^{n}-1\right)=(\alpha(G[H \backslash W], c)-d)+d-\alpha(G[H \backslash W], c)=0 .
$$

Finally, since $x^{n} \notin F_{W}, x^{1}, \ldots, x^{n}$ are affinely independent vectors in $\{x \in S T A B(G) \mid f(x)=$ $0\}$. 
The lifting operation in [17 corresponds to a special case of Lemma 3 in which inequality $c^{\top} x \leq d$ is a rank inequality of a projected graph's clique with empty intersection with $W$. More precisely, it includes the projected edge $u v$, a clique $\tilde{W}$ in the projected graph $\tilde{G}$, and $N(u) \cap N(v)$ such that $\tilde{W} \cap N(u) \cap N(v)=\emptyset, \tilde{W} \cap\{u, v\}=\emptyset$, and $N(u) \cap N(v)$ is a clique of $\tilde{G}$ to produce the valid inequality

$$
x_{\tilde{W}}+x_{\{u, v\}}+x_{N(u) \cap N(v)} \leq 2
$$

for $S T A B(G)$. It is straightforward to check that Lemma 3, with $W=\{u, v\} \cup(N(u) \cap N(v))$, $c^{\top} x \leq d$ being the clique inequality of $\tilde{W}$, and $\lambda=-1$, establishes that (5) is a valid inequality for $\operatorname{STAB}(G)$.

The clique projection operation and the corresponding clique-lifting operations according to Lemma 3 lead to stronger inequalities than those that can be obtained with the edge projection method proposed in [17. As an illustration, consider the structure in Figure 2(a) and $W=\{d, e, f\}$. The projection of de in this graph adds the false edge $a c$, and if we then lift the clique $\{a, b, c\}$ of $G \mid$ de we get the rank inequality $x_{a}+x_{b}+x_{c}+x_{d}+x_{e}+x_{f} \leq 2$. The same inequality is obtained with Lemma 3 if we take as $c^{\top} x \leq d$ the clique inequality of $G+d e$ for $\{a, b, c\}$. Nevertheless, even in this simple example, there is an inequality that cannot be derived with the method in 17. If we take $\{a, b, c, f\}$ as the clique inducing set of vertices associated with $c^{\top} x \leq d$ in Lemma 3 , then we get $x_{a}+x_{b}+x_{c}+x_{d}+x_{e}+2 x_{f} \leq 2$ as a valid (indeed, facet-defining [5]) inequality for $S T A B(G)$.

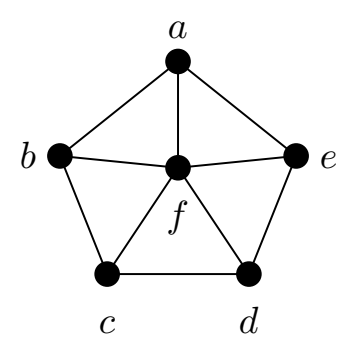

(a) Not rank inequality.

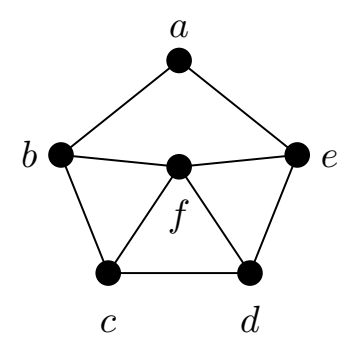

(b) Rank inequality.

Figure 2: Structures leading to stronger inequalities than edge projection.

The structure in Figure 2(b) (assuming that it induces a rank inequality of $G$ 2, 5]) also illustrates the fact that $\sum_{v \in W} x_{v} \leq 1$ being facet-defining for $S T A B(G)$ is not necessary to derive another facet of $S T A B(G)$. To show this, we choose $W=\{d, e\}$ and again take the clique inequality of $G+d e$ associated with $\{a, b, c, f\}$. With such a configuration, Lemma 3 gives the rank inequality $x_{a}+x_{b}+x_{c}+x_{d}+x_{e}+x_{f} \leq 2$ as well. Observe that this inequality is not derived by the method in [17] if edge ae is deleted before projecting de $\left(x_{b}+x_{c}+x_{d}+x_{e}+x_{f} \leq 2\right.$ would be generated instead).

\section{Two Procedures for Generating Valid Inequalities}

We are now in position of introducing the general cut-generating procedures based on the previous definitions and lemmas. We shall introduce two procedures. The first one is a simple procedure directly based on Lemma 3, whereas the second one is a strenghtening based on the results in 23. In both procedures, the generation of a valid inequality consists of the following two steps. 


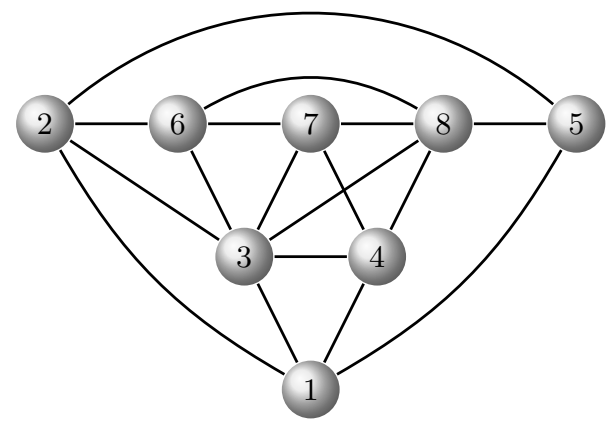

(a) $G_{0}$ (=G by definition).

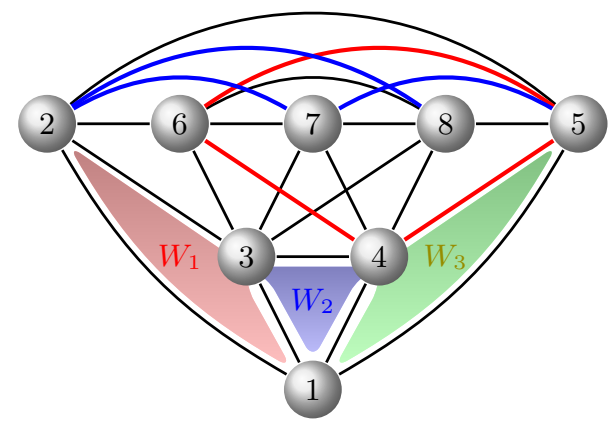

(b) $G_{3}$ (= $G_{2}$ in this example).

Figure 3: Projected graph $G_{3}$ (with false edges) is obtained after projecting $W_{1}=\{1,2,3\}, W_{2}=\{1,3,4\}$, and $W_{3}=\{1,4,5\}$, in this order.

Step 1: Sequence of clique projections. Define $G_{0}:=G$ and determine distinct subsets $W_{1}, \ldots, W_{r}$ of $V$ such that, for every $t \in\{1, \ldots, r\}, W_{t}$ is a clique of $G_{t-1}$ and $G_{t}$ is the projected graph $G_{t-1} \mid W_{t}$. An illustration of such a sequence is depicted in Figure 3 .

Step 2: Sequence of clique lifting operations. Let $W_{r+1} \subseteq V$ be a clique of $G_{r}$. The lifting procedure starts with the clique inequality $f_{r}(x)=x_{W_{r+1}} \leq 1$, which is valid for $S T A B\left(G_{r}\right)$, and iteratively for $t=r-1, \ldots, 0$ applies a specific version of Lemma 3 in order to generate $f_{t}(x)=f_{t+1}(x)+$ $\lambda_{t+1}\left(x_{W_{t+1}}-1\right) \leq 1$ in such a way that $f_{0}(x) \leq 1$ is valid for $S T A B\left(G_{0}=G\right)$.

\subsection{Basic Procedure}

The basic specific version of Lemma 3 , stated below, leads the general method to generate valid inequalities for all projected graphs $G_{0}, \ldots, G_{r}$. For $t \in\{1, \ldots, r\}$, let

$$
F_{W_{t}}=\left\{x \in S T A B\left(G_{t-1}\right) \mid x_{W_{t}}=1\right\} .
$$

Lemma 4. The inequality

$$
x_{W_{r+1}}+\sum_{t=1}^{r} \lambda_{t}^{B}\left(x_{W_{t}}-1\right) \leq 1
$$

is valid for $S T A B(G)$, where $P_{t}:=\left\{x \in S T A B\left(G_{t-1}\right) \mid x_{W_{t}}=0\right\}$ and

$$
\lambda_{t}^{B}:=\max \left\{x_{W_{r+1}}+\sum_{i=t+1}^{r} \lambda_{i}^{B}\left(x_{W_{i}}-1\right) \mid x \in P_{t}\right\}-1 .
$$

Proof. This result is obtained by iteratively applying Lemma 3 on $P_{t}$, for $t=r, \ldots, 1$ (i.e, in reverse order). At step $t$, the first inclusion of Lemma 1 assures that $x_{W_{t+1}} \leq 1$ is valid for $F_{W_{t+1}}$. Hence, being the lifting factor $\lambda_{t}^{B}$ calculated according to the definition in (4), the obtained inequality is valid. 
Consider the projected graph $G_{3}$ in Figure 3(b), and let $W_{4}=\{2,5,6,7,8\}$. The inequality $x_{W_{4}} \leq 1$ is trivially valid for $S T A B\left(G_{3}\right)$, and is also valid for $S T A B\left(G_{2}\right)$ since $\lambda_{3}^{B}=0$. This comes from $\lambda_{3}^{B}=\max \left\{x_{W_{4}} \mid x_{W_{3}}=0\right\}-1$, which has $\{8\}$ as an optimal solution. The remaining lifting operations are illustrated in Figure 4. finally giving rise to the inequality $x_{\{4,5,6,7,8\}}+2 x_{\{1,2,3\}} \leq 3$, which is valid for $S T A B(G)$.
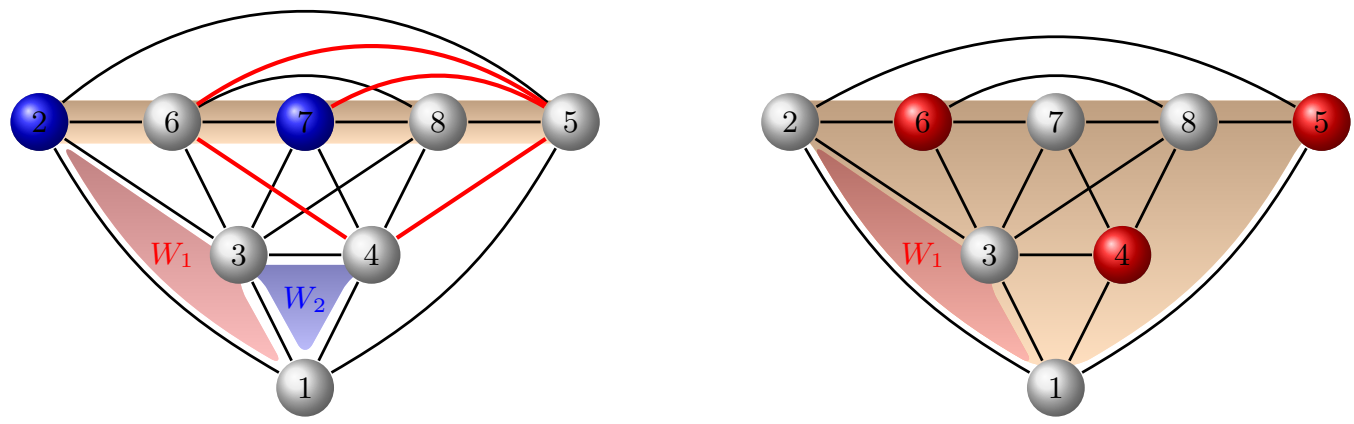

(a) $\operatorname{lifting} x_{W_{4}}$
$\max \left\{x_{W_{4}} \mid x_{W_{2}}=0\right\}$ $x_{W_{4}}+x_{W_{2}} \leq 2$, valid for $\operatorname{STAB}\left(G_{1}\right)$.

(b) The optimal solution $\{4,5,6\} \quad$ of $\max \left\{x_{W_{4}}+x_{W_{2}} \mid x_{W_{1}}=0\right\}$ yields $\lambda_{1}^{B}=$

Figure 4: Basic lifting procedure starting with the clique inequality of $W_{4}=\{2,5,6,7,8\}$ to generate $x_{\{4,5,6,7,8\}}+$ $2 x_{\{1,2,3\}} \leq 3$.

\subsection{Strengthened Procedure}

Let $F_{0}:=S T A B(G)$ and, for $t \in\{1, \ldots, r\}$,

$$
F_{t}=\left\{x \in F_{t-1} \mid x_{W_{t}}=1\right\}=\left\{x \in S T A B(G) \mid x_{W_{j}}=1, j=1, \ldots, t\right\} .
$$

Clearly, the integral elements of $F_{t}$ are stable sets of $G$. A property of the clique projection operation is that they are also stable sets of $G_{t}$. Define $E_{t}$ as the edge set of $G_{t}$, for $t=1, \ldots, r$.

Lemma 5. $F_{t} \subseteq S T A B\left(G_{t}\right)$, for all $t \in\{0, \ldots, r\}$.

Proof. We use induction on $t$ to show that $F_{t} \cap \mathcal{S}(G) \subseteq S T A B\left(G_{t}\right) \cap \mathcal{S}(G)$. The case $t=0$ is trivial. For $t \geq 1, F_{t-1} \subseteq S T A B\left(G_{t-1}\right)$ by the induction hypothesis and, consequently, $F_{t}=F_{t-1} \cap F_{W_{t}}$. The results follow from the first inclusion of Lemma 1.

By definition, $x_{W_{t}} \leq 1$ is valid for $S T A B\left(G_{t-1}\right)$. The previous lemma implies that it is also valid for the stable sets of $G$ that intersect $W_{1}, \ldots, W_{t-1}$.

Corollary 1. $x_{W_{t}} \leq 1$ is valid for $F_{t-1}$.

Our strengthened lifting procedure is as follows. We assume that $\max \emptyset=0$.

Lemma 6. Let $c^{\top} x \leq d, c, x \in \mathbb{R}^{n}$ and $d \in \mathbb{R}$, be a valid inequality for $S T A B\left(G_{r}\right)$. Then, $f_{t}(x) \leq d$ is valid for $F_{t}$, where, for $t \in\{0, \ldots, r\}$,

$$
f_{t}(x)=c^{\top} x+\sum_{\ell=t+1}^{r} \lambda_{\ell}^{S}\left(x_{W_{\ell}}-1\right)
$$


$\mathcal{S}_{t}=\mathcal{S}(G) \cap F_{t}$, and

$$
\lambda_{\ell}^{S}=\max \left\{f_{\ell}(x)-d \mid x \in \mathcal{S}_{\ell-1}, x_{W_{\ell}}=0\right\} .
$$

Proof. We show that $f_{t}(x) \leq d$ is valid for $F_{t}$ by induction on $t$. For $t=r$, the result follows since $f_{r}(x)=c^{\top} x \leq d$ is valid for $S T A B\left(G_{r}\right)$ and $F_{r} \subseteq S T A B\left(G_{r}\right)$ by Lemma 5 For $t<r$, by induction hypothesis, $f_{t+1}(x) \leq d$ is valid for $F_{t+1}=\left\{x \in F_{t} \mid x_{W_{t+1}}=1\right\}$. Applying the inequality construction, we get $\lambda_{t+1}^{S}=0$ if $\left\{x \in \mathcal{S}_{t} \mid x_{W_{t+1}}=0\right\}=\emptyset$, and

$$
\lambda_{t+1}^{S}=\max \left\{c^{\top} x+\sum_{i=t+2}^{r} \lambda_{i}^{S}\left(x_{W_{i}}-1\right) \mid \begin{array}{l}
x \in \mathcal{S}_{t}, \\
x_{W_{t+1}}=0
\end{array}\right\}-d
$$

otherwise. We now apply Lemma 3 , considering that $x_{W_{t+1}} \leq 1$ is valid for $F_{t}$ by Corollary 1 and $f_{t+1}(x) \leq d$ is valid for $F_{t+1}$, and then obtain that $f_{t}(x) \leq d$ is valid for $F_{t}$.

Let us take Figure 5 as an example of a sequence of $r=3$ clique liftings of the projected graph depicted in Figure 3(b)] For $t=3$, lifting $f_{3}(x)=x_{W_{4}} \leq 1$ with $\lambda_{3}^{S}=-1$ generates $f_{2}(x)=x_{\{2,6,7,8\}}-x_{\{1,4\}}+1 \leq 1$, which is valid for $F_{2}=\left\{x \in S T A B(G) \mid x_{W_{1}}=x_{W_{2}}=1\right\}$. The iterative procedure of Lemma 6 may generate stronger valid inequalities than the basic procedure in Lemma 4. For instance, for the example in Figure $4, x_{\{4,5,6,7,8\}}+2 x_{\{1,2,3\}} \leq 3$ is a linear combination between $x_{\{1,2,4,6,7,8\}}+2 x_{\{3\}} \leq 2$ and the clique inequality $x_{\{1,2,5\}} \leq 1$.

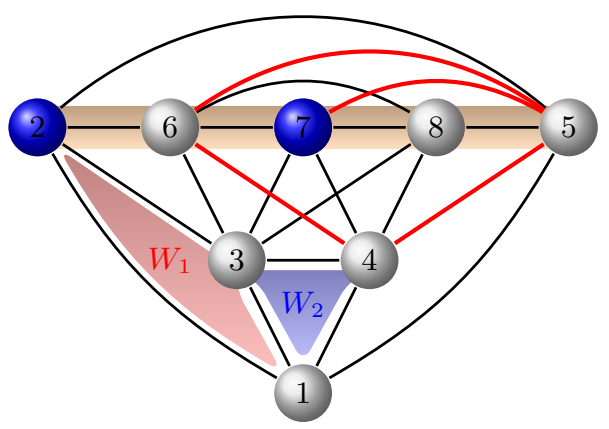

(a) Lifting $f_{2}(x) \leq 1$ with $\lambda_{2}^{S}=2$ gives $f_{1}(x)=$ $x_{\{1,2,4,6,7,8\}}+2 x_{\{3\}} \leq 2$, valid for $F_{1}=\{x \in$ $\left.S T A B(G) \mid x_{W_{1}}=1\right\}$.

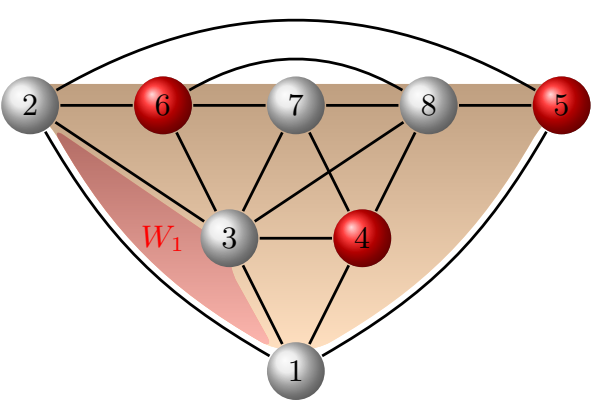

(b) $f_{0}(x)=f_{1}(x) \leq 2$ is also valid for $F_{0}$ since $\lambda_{1}^{S}=$ 0 .

Figure 5: Strengthened lifting procedure starting with the clique inequality of $W_{4}=\{2,5,6,7,8\}$ to generate $x_{\{1,2,4,6,7,8\}}+2 x_{\{3\}} \leq 2$.

\subsection{On the Strength of Lemma 6}

The following results state that the strengthened procedure is, in some sense, related to facetsubgraphs of $G$. The first result indicates that, in general, the inequality produced by the strengthened procedure is stronger than the one produced by the basic procedure. 
Lemma 7. Let $f_{0}(x) \leq 1$ be the inequality produced by the strengthened procedure. Then,

$$
1+\sum_{\ell=1}^{r} \lambda_{\ell}^{S}=\alpha\left(G\left[H^{S}\right], c^{S}\right) \leq \alpha\left(G\left[H^{B}\right], c^{B}\right) \leq 1+\sum_{\ell=1}^{r} \lambda_{\ell}^{B},
$$

where $H^{S} \subseteq \bigcup_{t=1}^{r+1} W_{t}$ and $H^{B} \supseteq H^{S}$ are the support, and $c^{S}$ and $c^{B}$ are the coefficient vectors, of $f_{0}(x) \leq 1$ and (6), respectively.

Proof. Let us examine the first equality. The inequality $1+\sum_{\ell=1}^{r} \lambda_{\ell}^{S} \geq \alpha\left(G\left[H^{S}\right], c^{S}\right)$ holds since $f_{0}(x) \leq 1$ is valid by Lemma 6 . For the converse inequality, a stable set of $H^{S}$ of weight $1+\sum_{\ell=1}^{r} \lambda_{\ell}^{S}$ can be constructed by including a subset of $W_{r+1}$ of weight $\lambda_{r}^{S}$ and a vertex of each $W_{t}$, for all $t \in\{0, \ldots, r-1\}$, by the definition of $F_{r-1}$ and $\lambda_{r}^{S}$.

The comparison between the basic and strengthened procedures is given by the next two inequalities. The former holds because $H^{S} \subseteq H^{B}$ and $\lambda_{t}^{B} \geq \lambda_{t}^{S}$, for all $t \in\{1, \ldots, r\}$, whereas the validity of (6) (by Lemma 4) implies the latter.

The second result establishes sufficient conditions for the generated inequalities to be facet defining. These conditions are slightly weaker than those in [23] due to two differences. First, the subsets $W_{3}, \ldots, W_{r}$ are not required to be cliques of $G$. Second, we use clique projection and we assume condition 3 to impose appropriate false edges in $G_{t^{\prime}}$ instead of the auxiliary contracted graph defined in 23] to determine $W_{r+1}$. Since the proof is very similar to the one in that paper, it is left to the appendix.

Theorem 1. If $f_{r}(x)=x_{W_{r+1}}$ and

1. $\left|W_{t}\right|=k$, the subgraph of $G_{t-1}$ induced by $\bigcup_{i=1}^{t} W_{i}$ is $k$-partite with vertex classes $V_{t}^{1}, \ldots, V_{t}^{k}$, and $W_{r+1}$ is a maximal clique of $G_{r}$ such that $W_{r+1} \cap V_{r}^{k}=\emptyset$,

2. $T_{t}:=\left(V_{t}, \mathcal{W}_{t}\right)$ is a strong hypertree defined by $V_{t}:=\bigcup_{i=1}^{k} V_{t}^{i}$ and $\mathcal{W}_{t}:=\left\{W_{1}, \ldots, W_{t}\right\}$. More precisely, either $\mathcal{W}_{t}=\left\{V_{t}\right\}$ or there is a $v \in V_{t}$ incident to a hyperedge $W_{i} \in \mathcal{W}_{t}$ sharing exactly $k-1$ vertices with some other hyperedge of $T_{t}$ such that $\left(V_{t} \backslash\{v\}, \mathcal{W}_{t} \backslash\left\{W_{i}\right\}\right)$ is also a strong hypertree,

3. for every $i \in\{1, \ldots, k-1\}$ and $w \in V_{r}^{0}$ such that $N_{G_{r}}(w) \cap V_{r}^{i} \neq \emptyset$, one of the following holds: $v \in W_{t} \cap V_{t}^{i}$ is a neighbor of $w$ in $G$ or there exists $t^{\prime} \in\{1, \ldots, r\}$ such that $W_{t}$ is a clique of $G_{t^{\prime}-1}, W_{t}$ and $W_{t^{\prime}}$ are adjacent in $T_{r}, v \notin W_{t^{\prime}}$, and $v^{\prime} \in W_{t^{\prime}} \cap V_{r}^{i}$ is a neighbor of $w$ in $G_{t^{\prime}-1}$,

4. no $v \in V_{t}^{k}$ has neighbors in $V_{r}^{0}$, i.e. $N_{G_{r}}(v) \cap V_{r}^{0}=\emptyset$,

hold for some $k>0$ and for all $t \in\{1, \ldots, r\}$, then $f_{t}(x) \leq 1$ is facet defining for $F_{t}$, for all $t \in\{1, \ldots, r\}$.

It can be noticed that the graph and the cliques $W_{1}, W_{2}, W_{3}, W_{4}$ in Figure 3 satisfy the sufficient conditions with $r=3$ and $k=3$. The above theorem implies that the inequality generated by the strengthened procedure (as shown in Figure 5 ) is indeed facet defining for the graph of the example. 


\section{The Separation Procedure}

We present in this section a separation procedure based on the valid inequality generation procedures presented in the last section. Algorithm 1 summarizes the proposed separation procedure. Besides the graph $G$, the input of this algorithm is a fractional solution $\bar{x}$ to be separated and a set $\mathcal{W}$ of maximal cliques of $G$. The variable $F$, initially empty, stores the set of violated inequalities that are generated by the separation procedure and returned at the end of its execution. For each clique $W_{1}$ in $\mathcal{W}$, we proceed by generating a sequence $S=\left\langle W_{1}, \ldots, W_{r+1}\right\rangle$ of distinct maximal cliques, with the corresponding sequence $\left\langle G_{0}, \ldots, G_{r}\right\rangle$ of projected graphs, and a set $T$ of indices $t$ such that the clique inequality associated with $W_{t}$ is violated for $S T A B\left(G_{t-1}\right)$. The generation of the sequence $S$ continues until a certain number of projections is performed and a violated clique inequality of the current projected graph is found. At this point, all subsequences $\left\langle W_{1}, \ldots, W_{t}\right\rangle$ of $S$ defined by a violated clique are lifted in reverse order as follows: for each $t \in T$, we apply Lemma 4 or Lemma 6 iteratively to $W_{t}$ in order to generate a valid inequality for the original graph. The computation of the lifting factors is accomplished with the algorithm in [15. The set of violated valid inequalities so generated (stored in $F$ ) is then returned.

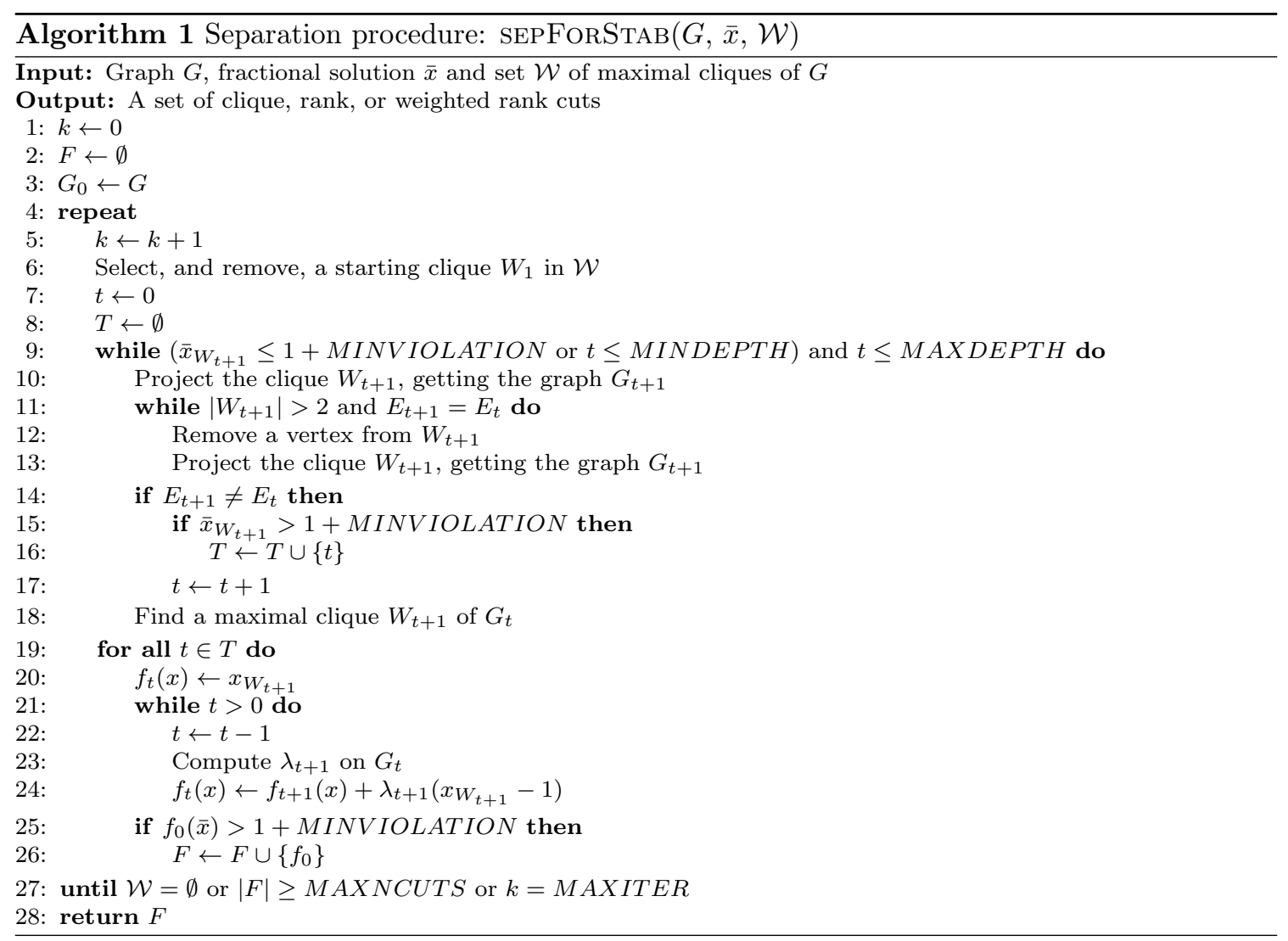

The execution of the separation procedure is governed by five parameters. Two parameters control the number of iterations $k$ of the main loop. According to the condition checked at line 27 
the loop is iterated at most MAXITER times, and this as long as there are cliques left in $\mathcal{W}$ and the number of violated inequalities encountered is at most MAXNCUTS. At each iteration, the size $r+1$ of the sequence of projections performed is at least MINDEPTH. The greater is the sequence size $r+1$, the larger is either the number of variables or the coefficients involved in the valid inequality generated after the lifting process. An inequality is considered violated only if its violation is greater than the threshold MINVIOLATION. An iteration fails if no violated clique inequality is found after $M I N D E P T H$ clique projections. The number of projections are bounded from above by parameter $M A X D E P T H$ because of possible failed iterations, which seldom occurs in practice.

The aim of the set $\mathcal{W}$ of maximal cliques given as input to the separation procedure is to yield different sequences of clique projections. The cliques in $\mathcal{W}$ are generated with two versions of a greedy algorithm. In both versions, the generation of a clique consists in selecting an initial vertex $v$ and then determining a maximal clique in the subgraph induced by $N(v)$. This clique in $N(v)$ is greedily built by considering the vertices sorted in a certain order. In one version, vertices are sorted in a nonincreasing order of weight, where the weight of a vertex $v$ is the value $\bar{x}_{\{v\}}$. A clique built with this version tends to have a relatively large intersection with previous cliques. In the other version, vertices not covered by previous cliques in $\mathcal{W}$ have priority with the purpose of generating cliques with small intersections. In order to avoid repetitions of cliques, the initial vertex of both versions is one not covered by previous cliques. In order to maintain a good probability of generating valid inequalities violated by $\bar{x}$, only cliques $W$ with $\bar{x}_{W} \geq 0.65$ are kept in $\mathcal{W}$.

Some remarks with respect to the generation of maximal cliques at line 18 are the following. The heuristic used to generate the maximal clique $W_{t+1}$ is similar to the one used to generate the cliques in $\mathcal{W}$. There are two differences, though: $W_{t+1}$ is guaranteed to include both a vertex that does not appear in $W_{0}, \ldots, W_{t}$ and a false edge in $E_{t} \backslash E_{t-1}$, when $t>0$. For every $K$ clique projections, we employ the algorithm in 21 to search for a violated clique. We do not generate all cliques, but stop when a prespecified number of cliques is enumerated instead. It might be the case that $G_{t+1}$ contains no false edges, relative to $G_{t}$, which means that no false edges are generated by the clique projection of $W_{t+1}$. In such a situation, vertices are iteratively removed from $W_{t+1}$ until either a false edge is generated in the projection of $W_{t+1}$ or $W_{t+1}$ has only one vertex. In the latter case, $W_{t+1}$ is discarded.

\section{Computational Experiments and Analysis of Results}

In this section we provide some results of computational experiments conducted in order to explore whether the proposed method is useful as a cut-generating tool for the MSS problem. Our main goal is not to provide a competitive algorithm for the MSS problem, since combinatorial algorithms are much more effective than cutting-plane algorithms for this problem [7, 19]. As already pointed out in [17, the facts that other combinatorial problems may be formulated including stable set constraints, either explicitly or devised to address their vertex packing relaxation, are motivations to the investigation of efficient polyhedral methods for the stable set problem. In this context, we intend to assess whether the proposed procedure is effective at generating rank or weighted rank cuts for the STAB polytope, and the nature of the obtained cuts. To this end, we performed three cutting-plane method implementations attached to the COIN-CLP linear programming solver to compute a strengthened upper bound for the MSS problem [9. In these implementations, a clique cover of all edges in $E$ is first determined and the corresponding inequalities constitute the initial 
model. The method consists in iteratively solving the current model. Whenever a fractional solution is found, we first select the set $\mathcal{W}$ of maximal cliques of $G$. The violated clique inequalities encountered in this process are added to the model. Then, the separation procedure of Algorithm 1 is executed. In addition to the separation procedure, we also implemented the rounding heuristic proposed in [16] and employed it to compute lower bounds.

The first implementation, called SFS_C, only employs the clique cuts found in the generation of $\mathcal{W}$ and aims to serve as a reference for evaluation of the two other implementations. These are implementations that include a call to SEPFORSTAB after the generation of $\mathcal{W}$. The difference between these two implementations is restricted to how the lifting operation at line 23 is performed. Version $S F S \_B$ uses the procedure established in Lemma 4 while Lemma 6 is employed in version $S F S \_S$. Various configurations of the parameters of SEPFORSTAB were tested and we chose to report the results corresponding to the following setup: MINVIOLATION $=0.03$, MINDEPTH $=10$, $K=10, M A X I T E R=50$, and MAXNCUTS $=20$. These were the values that produced the best average results. All the implementations were written in $\mathrm{C}++$, compiled with $\mathrm{g}^{++}-\mathrm{std}=\mathrm{c}^{++11}$ -m64 -0 -fPIC, and run on a Intel(R) Core(TM) i7-4790K CPU clocked at 4.00GHz.

Table 1 summarizes the results of experiments with some instances from the DIMACS benchmark and for random graphs with 100-300 vertices. The notation $G(n, d)$ specifies random graphs with $n$ vertices and a density of $d \in[0,1]$, and for these instances we report the average results over five randomly-generated instances. The experiments were performed on a 64 -bit personal computer, with a time limit of two minutes. The first four columns contain the instance name, the number of vertices, the graph density, and its stability number. The following columns contain data for the cutting-plane method: the column "LB" contains the lower bound found by the rounding heuristic, the columns "Upper bound" contain the upper bound obtained with the three implementations (in addition, the upper bound $Z_{0}$ corresponding to the linear relaxation of the initial model is also indicated), and the columns "Time" report the total time spent, in seconds.

We can observe the following facts from the data in Table 1. The graphs on which the reduction in the upper bound obtained with SEPFORSTAB is significant when compared to SFS_C are brock200_2, brock200_4, C125.9, C250.9 and DSJC500.5. For graphs c-fat200-5 and MANN_a45, clique cuts are not capable of improving the bound obtained with respect to $Z_{0}$. However, the rank and weighted rank cuts added with SEPForSTAB made versions $S F S \_B$ and $S F S \_S$ capable of improving the upper bound, attaining the optimal value in the first case. The only case where the versions $S F S \_B$ e $S F S \_S$ do not get better upper bounds than $S F S \_C$ is the graph san400_0.5_1. The reason for this phenomenon is that cliques become large in projected graphs at depth 6 and beyond, making the calculation of lifting factors very time consuming. Thus, the time limit is reached before violated inequalities are found. In Table 2, the results are presented with a depth limit of 3 projections, where we can observe the improvement of the bounds with respect to SFS_C. In general, there is a tendency of the version $S F S_{-} S$ to produce upper bounds slightly better than the version $S F S_{\_} B$. In particular, the graph DSJC500.5 is the case where the difference is more pronounced. Unlike the graphs with particular structures, random graphs present a homogenous behavior, with both versions $S F S_{-} B$ and $S F S_{-} S$ having better performance than $S F S_{-} C$, to the advantage of version $S F S \_S$. With regard to the comparison of the processing time between the versions with projection of cliques, we observed a trend to an increase in version $S F S \_S$ with respect to version $S F S \_B$. There are, however, 3 exceptions: p hat300-2, p hat300-3 and san200_0.7_2. In such cases, there is a significant reduction in processing time, with slight improvement in the upper bound obtained. This confirms the special case of gen400_p0.9_55 and gen400_p0.9_55 where the integer programming approach has performance far superior to combinatorial algorithms. The 


\begin{tabular}{|c|c|c|c|c|c|c|c|c|c|}
\hline \multicolumn{3}{|l|}{ Instance } & \multirow[b]{2}{*}{ LB } & \multicolumn{4}{|c|}{ Upper bound } & \multicolumn{2}{|c|}{ Time (sec.) } \\
\hline Graph & $n /$ Dens. & $\alpha$ & & $Z_{0}$ & SFS_C & $S F S_{-} B$ & $S F S_{-} S$ & $S F S_{-} B$ & $S F S_{-} S$ \\
\hline brock200_1 & $200 / 25$ & 21 & 20 & 45.48 & 38.57 & 35.18 & 34.85 & 41.76 & 44.86 \\
\hline brock200_2 & $200 / 50$ & 12 & 12 & 28.69 & 22.05 & 17.24 & 16.29 & 120.29 & 120.23 \\
\hline brock200_3 & $200 / 40$ & 15 & 14 & 35.66 & 28.21 & 24.22 & 23.26 & 120.22 & 120.47 \\
\hline brock200_4 & $200 / 34$ & 17 & 16 & 37.82 & 31.17 & 27.77 & 26.81 & 109.81 & 117.72 \\
\hline brock400_2 & $400 / 25$ & 12 & 24 & 78.95 & 65.68 & 63.85 & 63.27 & 120.67 & 120.95 \\
\hline brock400_4 & $400 / 25$ & 17 & 23 & 79.53 & 65.99 & 63.61 & 63.17 & 120.78 & 120.80 \\
\hline$c-f a t 200-1$ & $200 / 92$ & 12 & 12 & 12 & 12 & 12 & 12 & 0.04 & 0.04 \\
\hline$c-f a t 200-2$ & $200 / 84$ & 24 & 24 & 24 & 24 & 24 & 24 & 0.02 & 0.02 \\
\hline$c-f a t 200-5$ & $200 / 57$ & 58 & 58 & 66.66 & 66.66 & 58 & 58 & 22.67 & 27.10 \\
\hline c-fat500-1 & $500 / 96$ & 14 & 14 & 14 & 14 & 14 & 14 & 1.68 & 1.65 \\
\hline$c-f a t 500-10$ & $500 / 81$ & 126 & 126 & 126 & 126 & 126 & 126 & 0.97 & 0.97 \\
\hline$c-f a t 500-2$ & $500 / 93$ & 26 & 26 & 26 & 26 & 26 & 26 & 0.69 & 0.69 \\
\hline$c-f a t 500-5$ & $500 / 96$ & 64 & 64 & 64 & 64 & 64 & 64 & 0.81 & 0.81 \\
\hline C125. 9 & $125 / 10$ & 34 & 34 & 44.37 & 43.21 & 38.79 & 38.84 & 1.05 & 1.20 \\
\hline C250. 9 & $250 / 10$ & 44 & 43 & 77.25 & 71.78 & 65.71 & 65.35 & 11.88 & 18.04 \\
\hline DSJC125. 1 & $125 / 90$ & 34 & 34 & 45.28 & 43.22 & 39.23 & 39.41 & 1.21 & 0.94 \\
\hline DSJC125.5 & $125 / 50$ & 10 & 10 & 20.80 & 15.98 & 12.34 & 11.97 & 32.44 & 20.25 \\
\hline DSJC500.5 & $500 / 50$ & 13 & 13 & 58.94 & 46.16 & 39.44 & 35.54 & 123.20 & 123.29 \\
\hline gen400_p0.9_55 & $400 / 90$ & 55 & 55 & 93.07 & 55 & 55 & 55 & 1.09 & 1.22 \\
\hline gen400_p0.9_65 & $400 / 90$ & 65 & 65 & 103.59 & 65 & 65 & 65 & 1.32 & 1.36 \\
\hline gen400_p0.9_75 & $400 / 90$ & 75 & 75 & 106.54 & 75 & 75 & 75 & 2.59 & 2.84 \\
\hline hamming6-4 & $64 / 65$ & 4 & 4 & 7.57 & 5.33 & 4.28 & 4 & 0.24 & 0.23 \\
\hline hamming8-4 & $256 / 36$ & 16 & 16 & 16 & 16 & 16 & 16 & 0.18 & 0.16 \\
\hline keller4 & $171 / 35$ & 11 & 11 & 25.48 & 14.82 & 14.24 & 14.20 & 12.71 & 16.78 \\
\hline MANN_a27 & $378 / 1$ & 126 & 125 & 135 & 135 & 135 & 135 & 0.11 & 0.11 \\
\hline MANN_a45 & $1035 / 0.4$ & 345 & 341 & 363 & 363 & 360 & 360 & 1.39 & 1.43 \\
\hline MANN_a9 & $45 / 7$ & 16 & 16 & 18 & 18 & 18 & 18 & 0.00 & 0.00 \\
\hline p_hat300-1 & $300 / 75$ & 8 & 8 & 24.12 & 16.06 & 12.94 & 12.43 & 121.91 & 120.62 \\
\hline p_hat $300-2$ & $300 / 51$ & 25 & 25 & 45.34 & 34.06 & 33.64 & 33.50 & 37.18 & 30.29 \\
\hline p_hat $300-3$ & $300 / 26$ & 36 & 35 & 66.67 & 55.67 & 51.95 & 51.82 & 67.61 & 57.77 \\
\hline san200_0.7_2 & $200 / 30$ & 18 & 18 & 27.24 & 19.07 & 18.73 & 18.54 & 20.37 & 6.83 \\
\hline san200_0.9_1 & $200 / 10$ & 70 & 70 & 70.29 & 70 & 70 & 70 & 0.03 & 0.04 \\
\hline san200_0.9_2 & $200 / 10$ & 60 & 60 & 65.81 & 60 & 60 & 60 & 0.26 & 0.24 \\
\hline san200_0.9_3 & $200 / 10$ & 44 & 44 & 59.75 & 44 & 44 & 44 & 0.17 & 0.23 \\
\hline san400_0.5_1 & $400 / 50$ & 13 & 9 & 16.41 & 13.60 & 15.20 & 15.18 & 142.37 & 124.81 \\
\hline san400_0.9_1 & $400 / 10$ & 100 & 100 & 119.24 & 100.11 & 100 & 100 & 2.57 & 2.87 \\
\hline sanr200_0.7 & $200 / 30$ & 18 & 18 & 41.30 & 33.83 & 30.92 & 30.21 & 73.48 & 77.88 \\
\hline sanr200_0.9 & $200 / 10$ & 42 & 42 & 64.19 & 59.98 & 54.30 & 54.18 & 8.98 & 10.77 \\
\hline$\overline{G(100,10)}$ & $100 / 10$ & 31.2 & 31 & 37.56 & 36.25 & 33.07 & 33.06 & 0.35 & 0.38 \\
\hline$G(100,20)$ & $100 / 20$ & 20.2 & 20.2 & 29.28 & 26.53 & 23.87 & 23.86 & 1.65 & 1.67 \\
\hline$G(100,30)$ & $100 / 30$ & 15 & 15 & 24.91 & 21.04 & 18.45 & 18.23 & 3.69 & 4.58 \\
\hline$G(100,50)$ & $100 / 50$ & 9.2 & 9.2 & 17.19 & 13.45 & 10.83 & 10.60 & 7.82 & 6.52 \\
\hline$G(150,10)$ & $150 / 10$ & 37.4 & 37 & 51.51 & 48.56 & 44.07 & 43.99 & 2.17 & 2.60 \\
\hline$G(150,20)$ & $150 / 20$ & 22.4 & 22.4 & 39.84 & 35.37 & 32.20 & 32.06 & 8.10 & 10.35 \\
\hline$G(150,30)$ & $150 / 30$ & 16.6 & 16.6 & 32.88 & 27.80 & 24.57 & 24.19 & 23.49 & 25.22 \\
\hline$G(150,50)$ & $150 / 50$ & 10.2 & 10.2 & 23.14 & 18.06 & 14.15 & 13.53 & 59.00 & 50.63 \\
\hline$G(200,10)$ & $200 / 10$ & 41.6 & 41 & 63.99 & 60.86 & 55.11 & 55.04 & 7.12 & 8.07 \\
\hline$G(200,20)$ & $200 / 20$ & 26 & 25.4 & 50.73 & 44.56 & 40.87 & 40.65 & 23.76 & 30.26 \\
\hline$G(200,30)$ & $200 / 30$ & 18 & 17.8 & 40.75 & 34.35 & 31.21 & 30.49 & 60.53 & 84.12 \\
\hline$G(200,50)$ & $200 / 50$ & 11 & 11 & 29.13 & 22.32 & 17.62 & 16.65 & 115.00 & 112.76 \\
\hline$G(300,10)$ & $300 / 10$ & - & 44.8 & 90.70 & 82.81 & 75.81 & 75.91 & 33.74 & 35.91 \\
\hline$G(300,20)$ & $300 / 20$ & 28.4 & 27.2 & 69.84 & 59.97 & 56.55 & 56.38 & 74.90 & 84.56 \\
\hline$G(300,30)$ & $300 / 30$ & 20.2 & 19.6 & 56.79 & 47.20 & 43.93 & 43.31 & 120.60 & 120.66 \\
\hline$G(300,50)$ & $300 / 50$ & 12 & 12 & 40.07 & 30.68 & 24.47 & 22.90 & 121.00 & 121.29 \\
\hline
\end{tabular}

Table 1: Comparison of upper bounds and processing times among $S F S \_C, S F S \_B$, and $S F S \_S$. 
only combinatorial algorithm that solves these graphs is in [7.

\begin{tabular}{|c|c|c|c|c|c|c|c|c|c|}
\hline \multirow[b]{2}{*}{ LB } & \multicolumn{3}{|c|}{ Upper bound } & \multicolumn{2}{|l|}{ Time } & \multicolumn{2}{|c|}{$S F S_{-} B$ N. of cuts } & \multicolumn{2}{|c|}{$S F S_{-} S$ N. of cuts } \\
\hline & $S F S_{-} B$ & $S F S_{-} S$ & [16] & $S F S_{-} B$ & $S F S_{-} S$ & Rank & W-Rank & Rank & W-Rank \\
\hline 9 & 13.61 & 13.59 & 13.24 & 113.60 & 120.46 & 1437 & 1072 & 1331 & 1121 \\
\hline
\end{tabular}

Table 2: Behavior of $S F S_{-} B$ and $S F S \_S$ with graph san400_0.5_1 when the depth limit is 3.

As shown in Table 3 , the procedure is able to generate a large number of cuts, and provides upper bounds that are competitive with those generated in [16], [17, and [10] for a representative sample of benchmark graphs. It is worth remarking that the upper bound obtained with our approaches is tighter, in comparison the ones from the literature, when the density is between $40 \%$ and $75 \%$. The columns "Upper bound" contain the upper bound attained in [17, [16], and [10] in the root node of their branch-and-cut algorithms, respectively. Finally, the last three columns contain the number of generated clique cuts, violated rank inequalities, and violated weighted rank inequalities, respectively. Similarly to existing procedures, our cut-generating algorithm finds a large number of violated clique inequalities, and is also able to find many violated rank inequalities. The number of weighted rank inequalities generated by the procedure is smaller, but nevertheless provides an interesting set of additional and non-trivial valid inequalities. In Table 4 , similar results can be observed for random generated graphs.

Some characteristics of the results presented in Table 3 have been also observed in [16] in the context of a comparison between the approach adopted in that paper of combinining several separation heuristics and the one of edge projection of [17. It was observed that in some peculiar cases (notably, the sparse graphs C125.9 and C250.9), the edge projection alone performed better than the combination of cuts with respect to the upper bound obtained. The intriguing question that deserves further clarification is how a strategy which involves a number of approximations (for instance, in the removal of edges and in determining the righthand side of the cut resulting of the lifting operation) results in stronger cuts. An analysis of the description given in [17 indicates an inaccuracy in the proposed procedure that may generate non-valid inequalities, although the authors asserted in a personal communication that the cuts generated in the reported experiments are verified to be valid. This fact leaves the possibility of generating cuts that, though valid, are not generated by procedures that ensure the viability of all generated cuts. However, the cuts used in [17. can be considered as a reference of strong cuts for some sparse graphs. 


\begin{tabular}{|c|c|c|c|c|c|c|c|c|c|c|c|c|c|}
\hline \multicolumn{3}{|l|}{ Instance } & \multicolumn{5}{|c|}{ Upper bound } & \multicolumn{3}{|c|}{$S F S_{-} B:$ Number of cuts } & \multicolumn{3}{|c|}{$S F S_{-} S:$ Number of cuts } \\
\hline Graph & $n /$ Dens. & $\alpha$ & $S F S_{-} B$ & $S F S_{-} S$ & 16 & 17 & 10 & Clique & Rank & W-Rank & Clique & Rank & W-Rank \\
\hline brock200_1 & $200 / 25$ & 21 & 35.18 & 34.85 & - & & 33.59 & 849 & 728 & 2116 & 847 & $\overline{752}$ & 2083 \\
\hline brock200_2 & $200 / 50$ & 12 & 17.24 & 16.29 & 20.99 & 22.01 & 18.27 & 2957 & 591 & 6261 & 2893 & 628 & 6512 \\
\hline brock200_3 & $200 / 40$ & 15 & 24.22 & 23.26 & - & - & 23.55 & 1873 & 468 & 6065 & 2086 & 563 & 6115 \\
\hline brock200_4 & $200 / 34$ & 17 & 27.77 & 26.81 & 29.93 & 30.87 & 26.77 & 1491 & 531 & 5531 & 1568 & 622 & 5354 \\
\hline brock400_2 & $400 / 25$ & 12 & 63.85 & 63.27 & 63.84 & 67.66 & - & 2696 & 843 & 1394 & 2657 & 862 & 1686 \\
\hline brock400_4 & $400 / 25$ & 17 & 63.61 & 63.17 & 63.89 & 67.98 & - & 2685 & 778 & 1634 & 2706 & 937 & 1998 \\
\hline$c-f$ at $200-1$ & $200 / 92$ & 12 & 12 & 12 & 12.71 & 12.86 & - & - & - & - & - & - & - \\
\hline c-fat $200-2$ & $200 / 84$ & 24 & 24 & 24 & 24 & 24 & - & - & - & - & - & - & - \\
\hline$c-f a t 200-5$ & $200 / 57$ & 58 & 58 & 58 & 58.89 & 65.25 & 58 & 97 & 843 & 84 & 149 & 866 & 79 \\
\hline$c-f a t 500-1$ & $500 / 96$ & 14 & 14 & 14 & 14 & 14.98 & - & 107 & 13 & 9 & 72 & 15 & 11 \\
\hline$c-f$ at $500-10$ & $500 / 81$ & 126 & 126 & 126 & 126 & 223.29 & - & - & - & - & - & - & - \\
\hline$c-f$ at $500-2$ & $500 / 93$ & 26 & 26 & 26 & 26.97 & 57.78 & - & - & - & - & - & - & - \\
\hline$c-f a t 500-5$ & $500 / 96$ & 64 & 64 & 64 & 64.70 & 67.08 & - & - & - & - & - & - & - \\
\hline C125.9 & $125 / 10$ & 34 & 38.79 & 38.84 & 41.26 & 37.40 & 37.81 & 61 & 397 & 148 & 67 & 430 & 132 \\
\hline $\mathrm{C} 250.9$ & $250 / 10$ & 44 & 65.71 & 65.35 & 69.76 & 58.30 & 63.95 & 389 & 1111 & 399 & 380 & 1093 & 402 \\
\hline DSJC125.1 & $125 / 90$ & 34 & 39.23 & 39.41 & - & - & 38.22 & 55 & 387 & 149 & 51 & 365 & 147 \\
\hline DSJC125.5 & $125 / 50$ & 10 & 12.34 & 11.97 & - & - & 13.21 & 918 & 255 & 4182 & 968 & 261 & 3270 \\
\hline DSJC500.5 & $500 / 50$ & 13 & 39.44 & 35.54 & - & 52.95 & - & 5556 & 257 & 1066 & 5538 & 299 & 1291 \\
\hline gen400_p0.9_55 & $400 / 90$ & 55 & 55 & 55 & - & 56.20 & - & 513 & 254 & 33 & 523 & 257 & 32 \\
\hline gen400_p0.9_65 & $400 / 90$ & 65 & 65 & 65 & - & 65.25 & - & 598 & 246 & 23 & 620 & 269 & 17 \\
\hline gen400_p0.9_75 & $400 / 90$ & 75 & 75 & 75 & - & 75 & - & 866 & 408 & 55 & 901 & 475 & 55 \\
\hline hamming6-4 & $64 / 65$ & 4 & 4.28 & 4 & - & - & 4.64 & 291 & 120 & 383 & 310 & 164 & 417 \\
\hline hamming8-4 & $256 / 36$ & 16 & 16 & 16 & 16 & 16 & - & 173 & - & - & 173 & - & - \\
\hline keller4 & $171 / 35$ & 11 & 14.24 & 14.20 & 14.83 & 14.95 & 14.29 & 560 & 464 & 1076 & 542 & 535 & 1172 \\
\hline MANN_a27 & $378 / 1$ & 126 & 135 & 135 & - & 134.86 & 132.44 & - & - & - & - & - & - \\
\hline MANN_a45 & $1035 / 0.4$ & 345 & 360 & 360 & - & 360 & 355.86 & 17 & 22 & - & 17 & 22 & - \\
\hline MANN_a9 & $45 / 7$ & 16 & 18 & 18 & - & - & 17.11 & - & - & - & - & - & - \\
\hline p_hat $300-1$ & $300 / 75$ & 8 & 12.94 & 12.43 & - & - & 13.45 & 2851 & 141 & 4046 & 2703 & 174 & 4342 \\
\hline p_hat $300-2$ & $300 / 51$ & 25 & 33.64 & 33.50 & 33.81 & 34.19 & 30.73 & 923 & 89 & 1197 & 933 & 82 & 1153 \\
\hline p_hat 300-3 & $300 / 26$ & 36 & 51.95 & 51.82 & 54.12 & 53.19 & 49.79 & 995 & 764 & 1327 & 1086 & 759 & 1341 \\
\hline $\operatorname{san} 200 \_0.7 \_2$ & $200 / 30$ & 18 & 18.73 & 18.54 & 18.50 & 19.18 & 18 & 747 & 265 & 815 & 676 & 259 & 531 \\
\hline san200_0.9_1 & $200 / 10$ & 70 & 70 & 70 & 70 & 70 & - & 32 & 22 & 8 & 32 & 22 & 8 \\
\hline $\operatorname{san} 200 \_0.9 \_2$ & $200 / 10$ & 60 & 60 & 60 & 60 & 60 & - & 163 & 113 & 10 & 148 & 101 & 5 \\
\hline $\operatorname{san} 200 \_0.9 \_3$ & $200 / 10$ & 44 & 44 & 44 & 44 & 44.80 & - & 159 & 165 & 20 & 169 & 179 & 25 \\
\hline $\operatorname{san} 400 \_0.5 \_1$ & $400 / 50$ & 13 & 15.20 & 15.18 & 13.24 & 17.14 & - & 217 & 35 & 57 & 214 & 30 & 57 \\
\hline $\operatorname{san} 400 \_0.9 \_1$ & $400 / 10$ & 100 & 100 & 100 & 100 & 100.40 & - & 661 & 241 & 42 & 671 & 264 & 50 \\
\hline sanr200_0.7 & $200 / 30$ & 18 & 30.92 & 30.21 & - & - & 29.45 & 1196 & 593 & 3446 & 1272 & 642 & 3830 \\
\hline sanr200_0.9 & $200 / 10$ & 42 & 54.30 & 54.18 & - & - & 54.52 & 265 & 880 & 287 & 250 & 973 & 332 \\
\hline
\end{tabular}

Table 3: Upper bounds obtained with $S F S_{-} B$ and $S F S_{-} S$, and their comparison with the ones from [16], [17], and [10]. 


\begin{tabular}{|c|c|c|c|c|c|c|c|c|c|}
\hline \multicolumn{2}{|l|}{ Instance } & \multicolumn{2}{|c|}{ Upper bound } & \multicolumn{3}{|c|}{$S F S_{\_} B:$ Number of cuts } & \multicolumn{3}{|c|}{$S F S_{-} S:$ Number of cuts } \\
\hline Graph & $\alpha$ & $S F S_{-} B$ & $S F S_{-} S$ & Clique & Rank & W-Rank & Clique & Rank & W-Rank \\
\hline$G(100,10)$ & 31.2 & 33.07 & 33.06 & 35.2 & 300.4 & 83.4 & 34.2 & 297.4 & 93.2 \\
\hline$G(100,20)$ & 20.2 & 23.87 & 23.86 & 158.2 & 393.8 & 406.4 & 158 & 389.2 & 455 \\
\hline$G(100,30)$ & 15 & 18.45 & 18.23 & 295 & 289.4 & 1083.2 & 297 & 319.6 & 1073.2 \\
\hline$G(100,50)$ & 9.2 & 10.83 & 10.60 & 571 & 152.4 & 2066 & 556.4 & 156.6 & 1889.6 \\
\hline$G(150,10)$ & 37.4 & 44.07 & 43.99 & 117 & 559.4 & 217.8 & 117.6 & 573.2 & 224.2 \\
\hline$G(150,20)$ & 22.4 & 32.20 & 32.06 & 338.6 & 611.6 & 821.6 & 348.4 & 636.2 & 830.2 \\
\hline$G(150,30)$ & 16.6 & 24.57 & 24.19 & 684.2 & 465.6 & 2268.4 & 706.4 & 489.6 & 2294.2 \\
\hline$G(150,50)$ & 10.2 & 14.15 & 13.53 & 1322.8 & 348 & 5012 & 1305.8 & 350.8 & 4670.4 \\
\hline$G(200,10)$ & 41.6 & 55.11 & 55.04 & 242.8 & 858.4 & 320 & 235.2 & 878.6 & 329.2 \\
\hline$G(200,20)$ & 26 & 40.87 & 40.65 & 583.4 & 847.2 & 1089.2 & 592.2 & 886 & 1181.2 \\
\hline$G(200,30)$ & 18 & 31.21 & 30.49 & 1159.6 & 575 & 3255.4 & 1224 & 667.4 & 3824.6 \\
\hline$G(200,50)$ & 11 & 17.62 & 16.65 & 2861.8 & 558 & 6133.2 & 2856.8 & 560.2 & 6219.2 \\
\hline$G(300,10)$ & - & 75.81 & 75.91 & 520.8 & 1429.2 & 490.2 & 523.4 & 1458 & 480.2 \\
\hline$G(300,20)$ & 28.4 & 56.55 & 56.38 & 1228.4 & 1092.4 & 1223.4 & 1269 & 1120 & 1287.4 \\
\hline$G(300,30)$ & 20.2 & 43.93 & 43.31 & 2166.6 & 669 & 3903.2 & 2378.4 & 740.6 & 4041 \\
\hline$G(300,50)$ & 12 & 24.47 & 22.90 & 5550.8 & 610 & 3550.2 & 5541.4 & 666.2 & 3707.8 \\
\hline
\end{tabular}

Table 4: Upper bounds obtained and number of cuts applied with $S F S \_B$ and $S F S \_S$.

\section{Conclusions}

In this work we have presented general cut-generating procedures for the standard formulation of the maximum stable set polytope, which are able to generate both violated rank and generalized rank inequalities. The main objective of these procedures is to generalize existing ones based on edge projection, and employ a lifting procedure in order to construct general valid inequalities from an initial clique inequality by undoing the operation of clique projection in the original graph. The computational experiments show that the proposed procedures are effective at generating general cuts, and may be competitive in a general setting.

\section{References}

[1] A. Atamtürk, G. L. Nemhauser, and M. W. P. Savelsbergh. Conflict graphs in solving integer programming problems. European Journal of Operational Research, 121(1):40 - 55, 2000.

[2] E. Balas and M. Padberg. Set partitioning: a survey. SIAM Review, 18:710-760, 1976.

[3] I. M. Bomze, M. Budinich, P. M. Pardalos, and M. Pelillo. The maximum clique problem. In Handbook of Combinatorial Optimization, pages 1-74. Kluwer Academic Publishers, 1999.

[4] S. S. Brito, H. G. Santos, and M. Poggi. A computational study of conflict graphs and aggressive cut separation in integer programming. Electronic Notes in Discrete Mathematics, 50:355-360, 2015. Proc. of the VIII Latin-American Algorithms, Graphs and Optimization Symposium.

[5] M. Campêlo, V. Campos, and R. Corrêa. On the asymmetric representatives formulation for the vertex coloring problem. Discrete Applied Mathematics, 156(7):1097-1111, 2008.

[6] G. Cornuéjols. Valid inequalities for mixed integer linear programs. Mathematical Programming Ser. B, 112:3-44, 2008.

[7] R. C. Corrêa, P. Michelon, B. Le Cun, T. Mautor, and D. Delle Donne. A bit-parallel russian dolls search for a maximum cardinality clique in a graph. arxiv, abs/1407.1209, 2014. 
[8] I. Dukanovic and F. Rendl. Semidefinite programming relaxations for graph coloring and maximal clique problems. Mathematical Programming, pages 345-365, 2007.

[9] The COmputational INfrastructure for Operations Research Initiative. Coin-or linear programming. https://projects.coin-or.org/Clp.

[10] M. Giandomenico, F. Rossi, and S. Smriglio. Strong lift-and-project cutting planes for the stable set problem. Mathematical Programming Ser. A, 141(1-2):165-192, 2013.

[11] L. Lipták and L. Lovász. Critical facets of the stable set polytope. Combinatorica, pages 61-88, 2001.

[12] L. Lovász and M.D. Plummer. Matching theory. Annals of Discrete Mathematics, 33:544, 1986.

[13] L. Lovász and A. J. Schrijver. Cones of matrices and set-functions and 0-1 optimization. SIAM Journal on Optimization, 1:166-190, 1991.

[14] C. Mannino and A. Sassano. Edge projection and the maximum cardinality stable set problem. In DIMACS Ser. Discrete Math. Theoret. Comput. Sci., volume 26, pages 249-261.

[15] P. Östergård. A new algorithm for the maximum weight clique problem. Nordic Journal of Computing, 8(4):424-436, 2001.

[16] S. Rebennack, M. Oswald, D. O. Theis, H. Seitz, G. Reinelt, and P. M. Pardalos. A branch and cut solver for the maximum stable set problem. Journal of Combinatorial Optimization, 21:434-457, 2011.

[17] F. Rossi and S. Smriglio. A branch-and-cut algorithm for the maximum cardinality stable set problem. Operations Research Letters, 28:63-74, 2001.

[18] P. San Segundo, D. Rodríguez-Losada, and A. Jiménez. An exact bit-parallel algorithm for the maximum clique problem. Computers \&3 Operations Research, 38:571-581, 2011.

[19] P. San Segundo and C. Tapia. Relaxed approximate coloring in exact maximum clique search. Computers \& Operations Research, 44:185-192, 2014.

[20] E. Tomita and T. Kameda. An efficient branch-and-bound algorithm for finding a maximum clique with computational experiments. Journal of Global Optimization, 37(1):95-111, 2007.

[21] E. Tomita, A. Tanaka, and H. Takahashi. The worst-case time complexity for generating all maximal cliques and computational experiments. Theoretical Computer Science, 363:28 - 42, 2006 .

[22] Q. Wu and J.-K. Hao. A review on algorithms for maximum clique problems. European Journal of Operational Research, 242(3):693 - 709, 2015.

[23] A. S. Xavier and M. B. Campêlo. A new facet generating procedure for the stable set polytope. Electronic Notes in Discrete Mathematics, 37:183-188, 2011. 


\section{Appendix A. Sufficient Conditions for Faceteness}

Consider the subsets $W_{1}, \ldots, W_{r+1}$. We show in this section that if there exists $k>0$ such that the following conditions hold, for all $t \in\{1, \ldots, r\}$ :

(I) $\left|W_{t}\right|=k$ and the subgraph of $G_{t-1}$ induced by $\bigcup_{i=1}^{t} W_{i}$ is $k$-partite with vertex classes $V_{t}^{1}, \ldots, V_{t}^{k}$,

(II) $T_{t}:=\left(V_{t}, \mathcal{W}_{t}\right)$ is a strong hypertree defined by $V_{t}:=\bigcup_{i=1}^{k} V_{t}^{i}$ and $\mathcal{W}_{t}:=\left\{W_{1}, \ldots, W_{t}\right\}$,

(III) for all $w \in V_{t}^{0}:=V \backslash V_{t}$, there exists $i \in\{1, \ldots, k\}$ such that $N_{G_{t-1}}(w) \cap V_{t}^{i}=\emptyset$,

then

(i) $x_{W_{t}} \leq 1$ is facet defining for $F_{t-1}$.

If, in addition to conditions (II)-(II), we assume that

(IV) for every $i \in\{1, \ldots, k-1\}$ and $w \in V_{r}^{0}$ such that $N_{G_{r}}(w) \cap V_{r}^{i} \neq \emptyset$, one of the following holds: $v \in W_{t} \cap V_{t}^{i}$ is a neighbor of $w$ in $G$ or there exists $t^{\prime} \in\{1, \ldots, r\}$ such that $W_{t}$ is a clique of $G_{t^{\prime}-1}, W_{t}$ and $W_{t^{\prime}}$ are adjacent in $T_{r}, v \notin W_{t^{\prime}}$, and $v^{\prime} \in W_{t^{\prime}} \cap V_{r}^{i}$ is a neighbor of $w$ in $G_{t^{\prime}-1}$,

(V) no $v \in V_{t}^{k}$ has neighbors in $V_{r}^{0}$, i.e. $N_{G_{r}}(v) \cap V_{r}^{0}=\emptyset$,

then we prove that

(ii) $f_{t}(x) \leq 1$ is facet defining for $F_{t}$,

considering that $f_{r}(x)=x_{W_{r+1}}$ and $W_{r+1}$ is a maximal clique of $G_{r}$ such that $W_{r+1} \cap V_{r}^{k}=\emptyset$.

Appendix A.1. Proof of (i)

The proof of $(i)$ depends on the dimension of $F_{t}$, established next, which in turn depends on conditions (I)-(III).

Lemma 8. If (II) holds, then $\left|W_{\ell} \cap V_{t}^{i}\right|=1$, for all $\ell \in\{1, \ldots, t\}$ and $i \in\{1, \ldots, k\}$.

Proof. Since $W_{\ell}$ is a clique and $V_{t}^{i}$ is a stable set, $\left|W_{\ell} \cap V_{t}^{i}\right| \leq 1$. By condition (I), $\left|W_{\ell}\right|=k$ and there are at least $k$ stable sets intersecting $W_{\ell}$. Therefore, $\left|W_{\ell} \cap V_{t}^{i}\right| \geq 1$.

Lemma 9 (Adapted from Lemma 3.1 of [23]). If (I) -(III) hold, then $\operatorname{dim}\left(F_{t}\right)=n-t$.

Proof. Because the incidence matrix of $T_{t}$ has rank $t$ due to conditions (II), it follows that $\operatorname{dim}\left(F_{t}\right) \leq$ $n-t$. To prove that $\operatorname{dim}\left(F_{t}\right) \geq n-t$, we exhibit $n-t+1$ affinely independent vectors of $F_{t}$. For this purpose, define $x^{i}$ to be the incidence vector of $V_{t}^{i}$, for every $i \in\{1, \ldots, k\}$. Clearly, $x^{i} \in S T A B(G)$ and, by Lemma 8. $x_{W_{\ell}}^{i}=1$ for all $\ell \in\{1, \ldots, t\}$, which means that $x^{i} \in F_{t}$. For every $v \in V_{t}^{0}$, define $y_{v}=x^{i}+e_{v}$ where $i \in\{1, \ldots, k\}$ is such that $v$ is not adjacent to any vertex in $V_{t}^{i}$, by condition (III), and $e_{v}$ is the incidence vector of $\{v\}$. Again, it is easy to see that $y_{v} \in F_{t}$. The $\left|V_{t}^{0}\right|+k=n-(k+t-1)+k=n-t+1$ points $\left\{x^{i}\right\}_{i=1}^{k} \cup\left\{y_{v}\right\}_{v \in V_{t}^{0}}$ are affinely independent.

Theorem 2. If (I) (III) hold, then (i) holds for all $t \in\{1, \ldots, r\}$. 
Proof. By Lemma $9 \operatorname{dim}\left(F_{t}\right)=\operatorname{dim}\left(F_{t-1}\right)-1$. Thus, by Corollary $1 . F_{t}=\left\{x \in F_{t-1} \mid x_{W_{t}}=1\right\}$ is a facet of $F_{t-1}$.

The reader might observe that conditions (I)-(III) are sufficient for (ii) when $t<r$.

Theorem 3. If (I)-(III) hold for all $t \in\{1, \ldots, r\}, f_{r}(x)=x_{W_{r}}$, and $d=1$, then (ii) holds for all $t \in\{1, \ldots, r-1\}$.

Proof. By induction on $t$. For $t=r, x_{W_{r+1}} \leq 1$ is facet defining for $F_{r}$ by (it). For $t<r, x_{W_{t+1}} \leq 1$ is facet defining for $F_{t}$ by $(i)$ and $f_{t+1}(x)$ is facet defining for $F_{t+1}=\left\{x \in F_{t} \mid x_{W_{t+1}}=1\right\}$ by induction hypothesis. Thus, the result follows by Lemma 3 .

Appendix A.2. Proof of (ii)

Conditions (II)-(II) imply the following property of $G_{r}$ and the stable sets of $G$ that cover $W_{1}, \ldots, W_{r}$. A strong hyperpath is a strong hypertree with exactly two vertices of degree 1.

Lemma 10 (Adapted from Lemma 3.2 of [23]). If (I)-(II) hold and $x \in F_{t}$, then $x_{\{u\}}=x_{\{v\}}$, for all $i \in\{1, \ldots, k\}$ and $\{u, v\} \subseteq V_{t}^{i}$.

Proof. Considering that conditions (I) (II) hold, let $W_{t_{1}}, \ldots, W_{t_{q}}$ be the strong hyperpath in $T_{t}$ connecting $u, v$. We prove the result by induction on $q$. If $q=2$, then $x_{W_{t_{1}}}-x_{W_{t_{2}}}=x_{\{u\}}-x_{\{v\}}=0$. Otherwise, $q>2$. Let $w \in W_{t_{2}} \backslash W_{t_{1}}$. Since $u \notin W_{t_{2}}$, we conclude that $w \in V_{t_{2}}^{i}$ by Lemma 8 . Hence, $W_{t_{1}}, W_{t_{2}}$ is a strong hyperpath with 2 hyperedges connecting $u, w$, which gives $x_{\{u\}}=$ $x_{\{w\}}$. Moreover, $W_{t_{p}}, \ldots, W_{t_{q}}$, for $p=\max \left\{j \mid w \in W_{t_{j}}\right\}$, is a strong hyperpath with less than $q$ hyperedges connecting two vertices of $V_{t}^{i}$. By inductive hypothesis, $x_{\{w\}}=x_{\{v\}}$. Therefore, $x_{\{u\}}=x_{\{v\}}$.

Differently from [23, we determine $W_{r+1}$ in $G_{r}$ (instead of defining an auxiliary graph). For this purpose, we use the following property of $G_{r}$ due to conditions (I)-(II) and (IV).

Lemma 11. Let $v \in V_{t}^{i} \cap W_{t}$, for some $i \in\{1, \ldots, k-1\}$, and $w \in V_{r}^{0}$ be such that $V_{r}^{i} \cap N_{G_{r}}(w) \neq \emptyset$. If (I) -(II) and (IV) hold, then $v w \in E_{r}$.

Proof. If $v w \in E$, then the lemma is trivially valid. Otherwise, by condition (IV), let $t^{\prime} \in\{1, \ldots, r\}$ be such that $W_{t}$ is a clique of $G_{t^{\prime}-1}, W_{t}$ and $W_{t^{\prime}}$ are adjacent in $T_{r}$ (considering conditions (I) -(II)), $v \notin W_{t^{\prime}}$ and $v^{\prime} \in W_{t^{\prime}} \cap V_{r}^{i}$ is a neighbor of $w$ in $G_{t^{\prime}-1}$. In this situation, $W_{t^{\prime}} \subseteq N_{G_{t^{\prime}-1}}(v) \cup N_{G_{t^{\prime}-1}}(w)$ implies $v w \in E_{t^{\prime}} \subseteq E_{r}$ by the clique projection of $W_{t^{\prime}}$.

To show (ii), we still need the following property of the subgraph of $G_{r}$ induced by $V_{r}^{0}$ and a certain subset of vertices. Notation $\cong$ denotes the affine isomorphism relation 23].

Lemma 12 (Adapted from Lemma 3.3 of 23]). If (I) -(V) hold for $t=r$, then $F_{r} \cong S T A B\left(G_{r}\left[V_{r}^{0} \cup\right.\right.$ $R]$ ) where $R \subseteq V$ is such that $\left|R \cap V_{r}^{i}\right|=1$, for all $i \in\{1, \ldots, k-1\}$, and $R \cap V_{r}^{k}=\emptyset$.

Proof. In what follows, we denote by $v_{i}$ the unique vertex in $R \cap V_{r}^{i}$, for all $i \in\{1, \ldots, k-1\}$.

$S T A B\left(G_{r}\left[V_{r}^{0} \cup R\right]\right) \rightarrow F_{r}$ : Take a point in $y \in S T A B\left(G_{r}\left[V_{r}^{0} \cup R\right]\right)$. For each $u \in V$, set $x_{u}=y_{u}$ if $u \in V_{r}^{0} ; x_{u}=y_{v_{i}}$, if $u \in V_{r}^{i}, i \in\{1, \ldots, k-1\}$; and $x_{u}=1-\sum_{i=1}^{k-1} y_{v_{i}}$, if $u \in V_{r}^{k}$. We prove that $x \in F_{r}$. First, to show that $x \in S T A B(G)$, take $u w \in E$. If $u, w \in V_{r}^{0} \cup R$, then $x_{u}+x_{w} \leq 1$ trivially holds. If $u, w \in V_{r}$, then $x_{u}+x_{w} \leq 1$ because $\{u, w\} \backslash V_{r}^{i} \neq \emptyset$, for all 
$i \in\{1, \ldots, k\}$. Otherwise, assume without loss of generality that $u \in V_{r} \backslash R$ and $w \in V_{r}^{0} \backslash R$. It turns out that $u \notin V_{r}^{k}$ by condition $(\mathrm{V})$. Then, use Lemma 11 to conclude that $v_{i} w \in E_{r}$ and, consequently, $x_{u}+x_{w} \leq 1$. To show that $x_{W_{\ell}}=1$, for $\ell \in\{1, \ldots, t\}$, we use Lemma 8 to write $x_{W_{\ell}}=\sum_{i=1}^{k-1} y_{v_{i}}+\left(1-\sum_{i=1}^{k-1} y_{v_{i}}\right)=1$.

$F_{r} \rightarrow S T A B\left(G_{r}\left[V_{r}^{0} \cup R\right]\right):$ Take $x \in F_{r}$. For each $v \in V_{r}^{0}$, set $y_{v}=x_{v}$, and for each $i \in\{1, \ldots, k-$ 1 \}, set $y_{v_{i}}=x_{v_{i}}$. This mapping is injective due to Lemma 10. Take $v w \in E\left[V_{r}^{0} \cup R\right]$. It is straightforward to check that $y_{v}+y_{w} \leq 1$.

Claim (ii) follows directly from Lemma 12 combined with the Lifting Lemma, as follows.

Theorem 4. If (I) $-(\mathrm{II})$ and $(\mathrm{IV})-(\mathrm{V})$ hold, $f_{r}(x)=x_{W_{r+1}}$, and $W_{r+1}$ is a maximal clique of $G_{r}$ such that $W_{r+1} \cap V_{r}^{k}=\emptyset$, then (ii) holds for all $t \in\{1, \ldots, r\}$.

Proof. By induction on $t$. For $t=r, x_{W_{r+1}} \leq 1$ is facet defining for $S T A B\left(G_{r}\left[V_{r}^{0} \cup R\right]\right)$, for every $R \subseteq V$ such that $\left|R \cap V_{r}^{i}\right|=1$, for all $i \in\{1, \ldots, k-1\}, R \cap V_{r}^{k}=\emptyset$, and $W_{r+1} \subseteq R$. Such an $R$ exists since $W_{r+1} \cap V_{r}^{k}=\emptyset$. Hence, by Lemma 12, $f_{r}(x)=x_{W_{r+1}} \leq 1$ is facet defining for $F_{r}$. For $t<r, x_{W_{t+1}} \leq 1$ is facet defining for $F_{t}$ by (i) (notice that (V) implies (III) and therefore we can use Theorem 2) and $f_{t+1}(x)$ is facet defining for $F_{t+1}=\left\{x \in F_{t} \mid x_{W_{t+1}}=1\right\}$ by induction hypothesis. Thus, the result follows by Lemma 3 . 